\title{
Novel Insights into Acid-Sensing Ion Channels: Implications for Degenerative Diseases
}

\author{
Ren-Peng Zhou ${ }^{1,2}$, Xiao-Shan Wu ${ }^{1,2}$, Zhi-Sen Wang ${ }^{1,2}$, Ya-Ya Xie ${ }^{1,2}$, Jin-Fang Ge ${ }^{1,2}$, Fei-Hu \\ Chen ${ }^{1,2, *}$ \\ ${ }^{1}$ Anhui Key Laboratory of Bioactivity of Natural Products, School of Pharmacy, Anhui Medical \\ University, Hefei 230032, China \\ ${ }^{2}$ The Key Laboratory of Anti-inflammatory and Immune Medicine, Anhui Medical University, Ministry \\ of Education, Hefei 230032, China
}

[Received October 30, 2015; Revised December 12, 2015; Accepted December 13, 2015]

\begin{abstract}
Degenerative diseases often strike older adults and are characterized by progressive deterioration of cells, eventually leading to tissue and organ degeneration for which limited effective treatment options are currently available. Acid-sensing ion channels (ASICs), a family of extracellular $\mathrm{H}^{+}-$ activated ligand-gated ion channels, play critical roles in physiological and pathological conditions. Aberrant activation of ASICs is reported to regulate cell apoptosis, differentiation and autophagy. Accumulating evidence has highlighted a dramatic increase and activation of ASICs in degenerative disorders, including multiple sclerosis, Parkinson's disease, Huntington's disease, intervertebral disc degeneration and arthritis. In this review, we have comprehensively discussed the critical roles of ASICs and their potential utility as therapeutic targets in degenerative diseases.
\end{abstract}

Key words: acid-sensing ion channel (asic), calcium $\left(\mathrm{ca}^{2+}\right)$, degenerative diseases, therapeutic target

Degenerative diseases are characterized by massive cell loss, ultimately leading to deterioration in quality or function of tissues or organs and possible failure of vital organs $[1,2]$. Although the etiology and pathogenesis of these diseases remain unclear, recent advances indicate that the processes of organ deterioration share common core features, including cell injury and dysfunction that contribute to functional and morphological impairment of cells. Despite considerable progress in understanding the molecular mechanisms of degenerative diseases, current therapeutic options are limited and no effective treatment drugs have emerged to date. Elucidation of both the common and unique mechanisms of deterioration may therefore facilitate the identification and development of effective anti-degenerative targets and drugs.

Acid-sensing ion channels (ASICs) belonging to the degenerin/epithelial sodium channel (DEG/ENaC) superfamily are widely distributed within mammalian nervous systems as well as non-neural tissues, such as cancer cells [3], articular chondrocytes [4] and intervertebral disc cells [5], where they play significant pathophysiological roles. ASICs are proton-gated cation channels activated by acidosis, lactate and arachidonic acid, which are involved in $\mathrm{Na}^{+}$and $\mathrm{Ca}^{2+}$ flux [6]. Intracellular $\mathrm{Ca}^{2+}\left(\left[\mathrm{Ca}^{2+}\right]_{\mathrm{i}}\right)$ is a ubiquitous second messenger in signal transduction pathways that modulates diverse physiological functions. Under pathological conditions, a robust increase in $\left[\mathrm{Ca}^{2+}\right]_{i}$ usually occurs through various extracellular $\mathrm{Ca}^{2+}$ influx and intracellular $\mathrm{Ca}^{2+}$ release mechanisms [7]. $\mathrm{Ca}^{2+}$ influx into cells is commonly mediated through activating channels or receptors, such as voltage-gated $\mathrm{Ca}^{2+}$ channels, $\alpha$-amino-

*Correspondence should be addressed to: Dr. Fei-Hu Chen, School of Pharmacy, Anhui Medical University, 81 Meishan Road, Hefei, Anhui Province, China 230032. Email: cfhchina@,sohu.com.

Copyright: ( 2016 Zhou RP, et al. This is an open-access article distributed under the terms of the Creative Commons Attribution License, which permits unrestricted use, distribution, and reproduction in any medium, provided the original author and source are credited. 
3-hydroxyl-5-methyl-4-isoxazole-propionate receptors, transient receptor potential channels, and $N$-methyl-Daspartate receptors [8]. Interestingly, ASICs have been shown to play crucial roles in modulating cell behavior via regulation of intracellular $\mathrm{Ca}^{2+}$ accumulation, including apoptosis [4, 9], differentiation [10], and autophagy [11]. Multiple lines of evidence suggest that aberrant expression and activation of ASICs contribute to the progression of various degenerative diseases, including multiple sclerosis, Parkinson's disease, Huntington's disease, intervertebral disc degeneration and arthritis. This review provides a summary of the properties of ASICs and their functional roles in the degenerative processes of several diseases, with further focus on their potential utility as novel pharmacological and therapeutic targets for degenerative diseases.

\section{Structure and characteristics of ASICs}

ASICs are voltage-independent, proton-gated cation channels that can be blocked by amiloride. To date, at least seven different ASIC isoforms (ASIC1a, ASIC1b,
$\mathrm{ASIC}_{1} \mathrm{~b}_{2}$, ASIC2a, ASIC2b, ASIC3, ASIC4) encoded by four separate genes (Accn1, Accn2, Accn3 and Accn4) have been identified in mammals [12-14]. All members of the ASIC family share the same topology as the $\mathrm{DEG} / \mathrm{ENaC}$ family, comprising two hydrophobic transmembrane domains (TM1 and TM2), short intracellular $\mathrm{N}$-and $\mathrm{C}$ - termini, and a large cysteine-rich extracellular loop [15, 16] (Fig.1A). The extracellular domain of ASICs has a highly negative cavity, designated 'acidic pocket', which is located distant from the transmembrane domain [17]. This acidic pocket, considered an ASIC $\mathrm{pH}$ sensor, contains several pairs of acidic amino acids and is responsible for acid-dependent gating, desensitization as well as response to specific extracellular modulators [18]. The functional channel of ASICs is a trimer of these subunits [17] (Fig.1B). The majority of homomeric and/or heteromeric trimers have different properties. Interestingly, however, ASIC2b and ASIC4 cannot form functional homomeric proton-gated channels by themselves $[19,20]$.

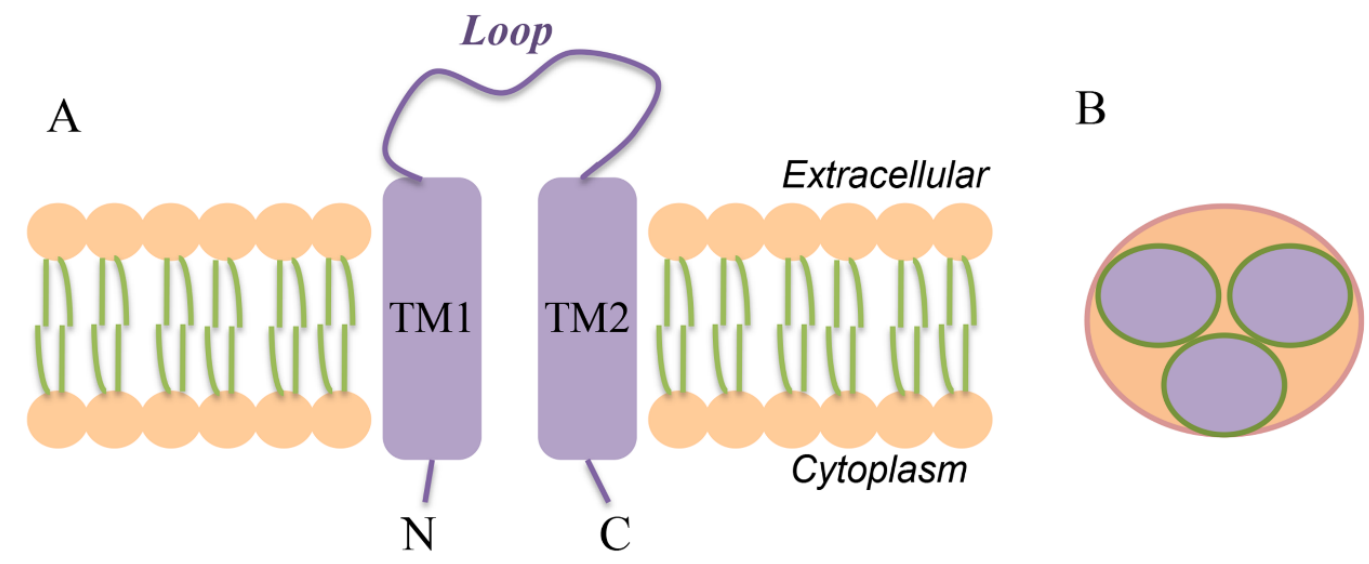

$\mathrm{C}$
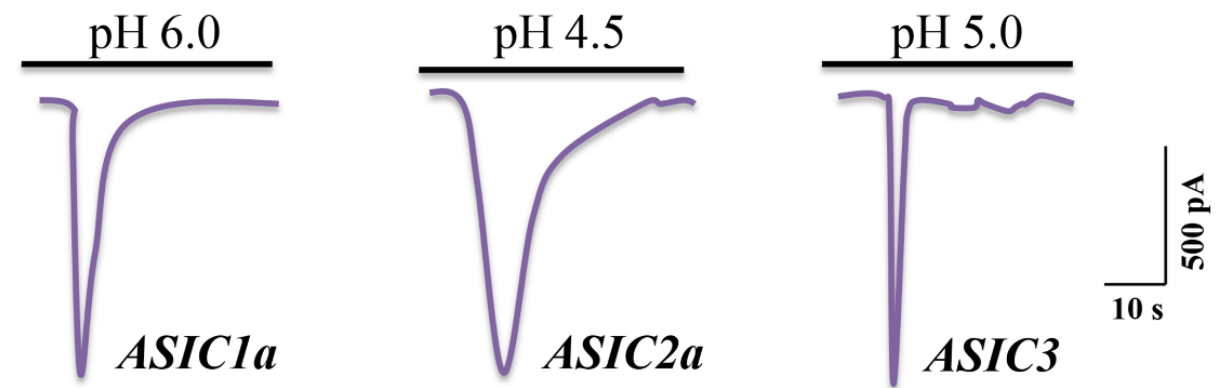

Figure 1. The structure and electrophysiological properties of ASICs. (A) Structure of individual ASIC subunits. (B) Three subunits assemble to form a functional homo- or heterotrimeric channel. (C) Electrophysiological properties of ASICs: representative traces of ASIC1a, ASIC2a, and ASIC3 in pH 6.0, 4.5, and 5.0 solutions, respectively. The membrane potential was clamped to $-60 \mathrm{mV}$. 
Table 1. Properties of ASIC channels

\begin{tabular}{|c|c|c|c|c|c|}
\hline Gene & Protein & Alternative name & pH50 activation & Inhibitor & References \\
\hline \multirow[t]{2}{*}{ Accn2 } & ASIC1a & $\begin{array}{c}\mathrm{ASIC} \alpha \\
\mathrm{BNaC} 2 \alpha\end{array}$ & $6.2-6.8$ & $\begin{array}{c}\text { Amiloride } \\
\text { PcTx1 } \\
\text { Mambalgins } \\
\text { Benzamil; } \\
\text { A-317567 }\end{array}$ & {$[6,15-17,21,36]$} \\
\hline & ASIC1b & $\begin{array}{c}\mathrm{ASIC} \beta \\
\mathrm{BNaC} 2 \beta\end{array}$ & 5.9 & $\begin{array}{l}\text { Amiloride } \\
\text { Mambalgins }\end{array}$ & \\
\hline \multirow[t]{2}{*}{ Accn1 } & ASIC2a & $\begin{array}{c}\text { MDEG1 } \\
\text { BNaC1 } \alpha \\
\text { BNC1 }\end{array}$ & 4.35 & $\begin{array}{l}\text { Amiloride } \\
\text { A-317567 }\end{array}$ & {$[15,22,38,39]$} \\
\hline & ASIC $2 b$ & $\begin{array}{l}\text { MDEG2 } \\
\text { BNaC1 } \beta\end{array}$ & N/A & N/A & \\
\hline Accn3 & ASIC3 & $\begin{array}{c}\text { DRASIC } \\
\text { TNaC }\end{array}$ & $6.2-6.7$ & $\begin{array}{l}\text { Amiloride } \\
\text { APETx2; } \\
\text { A-317567 }\end{array}$ & {$[15,36,43,44]$} \\
\hline Accn 4 & ASIC4 & SPASIC & N/A & N/A & [15] \\
\hline
\end{tabular}

N/A, not applicable

ASIC subunits are abundantly expressed in central and peripheral neurons and non-neural tissues [21], but show variable distribution. All the isoforms are expressed in the peripheral nervous system [22], while ASIC1a, ASIC2a and ASIC2b subunits are primarily localized in the central nervous system (CNS) [19, 23]. ASIC1a is widely expressed throughout the cerebral cortex, hippocampus, cerebellum, pineal gland, amygdala, dorsal root ganglion, and bone [24-27]. ASIC1b is almost exclusively expressed in sensory neurons. In contrast, ASIC3 is predominantly expressed in dorsal root ganglia neurons, especially nociceptive sensory neurons [28]. ASIC4, a new member of this ion channel group, exists within inner ear neurons, adenohypophysis, and intervertebral disc [29, 30].

ASICs are extremely susceptible to reduction of extracellular $\mathrm{pH}$. Under pathological conditions, such as inflammation, ischemia and hypoxia, decrease in extracellular $\mathrm{pH}$ from $\sim 7.5$ to 4 triggers activation of ligand-gated cation channels, including ASICs [31, 32]. Despite similar topological structures, different subunits display distinct sensitivities to additional decreases in extracellular $\mathrm{pH}$ (Fig.1C). For instance, ASIC1a and ASIC3 are the most sensitive to $\mathrm{H}^{+}$channel proteins of these subunits than other ASIC members, which are activated by $\mathrm{pH}$ levels below 7.0 [33]. In contrast to ASIC1a homomers, ASIC2a shows low sensitivity to reduced extracellular $\mathrm{pH}\left(\mathrm{pH}_{50}=4.35\right)$ and slow channel inactivation [34, 35]. Moreover, homomeric ASIC2a displays slower kinetics of desensitization than ASIC1a homomers $[36,37]$. ASIC $2 b$ does not form functional ion channels by itself, distinct from homomeric ASIC2a subunits [19]. On the other hand, ASIC2b associates with other ASIC subunits to form heteromultimeric channels with unique functional properties $[19,35]$. ASIC3 is primarily expressed in peripheral sensory neurons and plays an important role in pain perception, particularly high-intensity pain stimulation and acid-induced hyperalgesia [38]. The current of homomeric ASIC3 consists of instantaneous and steady-state components, which differ significantly in sensitivity to extracellular hydrogen ions [39]. The electrophysiological characteristics of ASIC4 remain largely unknown and require further study (Table 1).

\section{ASICs and Multiple Sclerosis}

Multiple sclerosis (MS) is a demyelinating autoimmune disease of the CNS affecting both the brain and spinal cord, which leads to axonal degeneration [40]. Although the etiology of MS is unclear, new insights suggest oligodendrocyte apoptosis as one of the critical events in physiological and pathophysiological processes [41]. Several studies have revealed that cytokine and ionic imbalance are the most important factors in axonal degeneration through inducing neuron mitochondrial dysfunction, alteration of ion exchange mechanisms and energy failure [42]. Recent histological analyses and in vivo studies have confirmed that undue accumulation of $\mathrm{Ca}^{2+}$ and $\mathrm{Na}^{+}$ions contributes to axonal degeneration during MS, and activation of ASIC1 plays a crucial role in accumulation of $\mathrm{Na}^{+}$and $\mathrm{Ca}^{2+}$ ions $[43,44]$. Disruption 
of the ASIC1 gene in mice markedly attenuated clinical deficits and axonal degeneration in an experimental autoimmune encephalomyelitis (EAE) mouse model of MS. Moreover, $\mathrm{pH}$ measurements showed that the $\mathrm{pH}$ dropped from $\sim 7.4$ to $\sim 6.5$ in inflammatory CNS lesions, indicating that tissue acidosis is sufficient to open the ASIC1 channel in the spinal cord of EAE mice. Inhibition of ASICs by the non-specific blocker, amiloride, led to neuroprotective effects against axonal degeneration [40]. Another recent study revealed enhanced expression of ASIC1 in spinal cord, optic nerve tissues and axons within lesions from patients with active MS and mice with acute EAE [45]. Increased ASIC1 expression was additionally observed via co-localization with the axonal damage marker, $\beta$-amyloid precursor protein, and associated with axonal injury. Remarkably, amiloride exerted protective effects against myelin and neuronal injury in the acute model, and ameliorated disability in mice with chronicrelapse EAE. In addition, 4-aminopyridine influenced the symptoms of MS as well as the course of the disease via inhibitory actions on ASIC and voltage-gated potassium channels [46]. These findings collectively support the potential efficacy of ASIC1 as a protective target for axon degeneration associated with active MS.

Consistent with the pivotal role of ASIC1 in the animal model of MS, studies by Arun et al. [47] showed that amiloride exerts a neuroprotective effect in patients with primary progressive MS. The normalized annual rate of whole-brain volume during the amiloride treatment phase (3 years) was significantly reduced, compared to the pretreatment phase. Similarly, changes in diffusion indices of tissue damage within primary clinically relevant deep grey matter and white matter structures were markedly alleviated during the treatment phase. A significant association between polymorphisms in MS and ASIC2 was revealed in a genome-wide study, further confirming the theory that ASIC2 is involved in the pathogenesis of MS [48]. Taken together, these findings suggest that blockade of ASICs may provide an alternative therapeutic approach to attenuate axon degeneration associated with MS. Nevertheless, the mechanisms by which ASICs regulate related cellular processes involved in MS, such as inflammation, remain to be established.

\section{ASICs and Parkinson's disease}

Parkinson's disease (PD) is a chronic, progressive neurodegenerative disease characterized by the degeneration of midbrain dopaminergic neurons, resulting in motor dysfunction and disability [49, 50]. The underlying mechanisms of neuronal loss associated with PD processes are currently unclear. A number of cell death pathways have been described in PD. Programmed cell death is a mechanism underlying cell demise in numerous pathologies, including progressive neurodegenerative disorders [51]. Midbrain dopamine neurons are vulnerable to toxic damage, which leads to disorders, such as PD. The pathologic process is associated with cerebral lactic acidosis. A previous report focused on the presence and characteristics of ASICs in mesolimbic dopamine neurons [52]. More recent studies have provided direct evidence that amiloride not only protects substantia nigra neurons from 1-methyl-4-phenyl-1,2,3,6-tetrahydropyridine-induced degeneration but also preserves dopaminergic cell bodies in the substantia nigra [53]. Additionally, administration of PcTX venom, a specific blocker of ASIC1a, had a modest effect, reducing loss in striatal dopamine active transporter binding and dopamine uptake. Interestingly, a deficit in the ubiquitin E3 ligase, parkin, significantly promoted the protein kinase Cevoked potentiation of native ASIC-like currents in hippocampal neurons. ASIC signaling may play a pivotal role in defects in parkin-mediated monoubiquitination of protein interacting with $\mathrm{C}$ kinase 1 that contribute to dopamine neuron degeneration in PD [54]. Paeoniflorin (PF), a monoterpene glycoside extracted from the root of Chinese herb Radix Paeoniaealba, is traditionally used to treat neurodegenerative disorders, especially PD. Both amiloride and PF protected PC12 cells against acidinduced injury and apoptosis by reducing $\mathrm{Ca}^{2+}$ influx, possibly through inhibition of ASIC1a channels [55]. Notably, the neuroprotective effects of amiloride and PF were associated with upregulation of autophagyrelated protein light chain 3 (LC3)-II. Furthermore, PF enhanced the autophagic degradation of $\alpha$-synuclein via modulating protein expression and activity of ASICs, subsequently leading to protective effects against acidosis-induced cytotoxicity [11]. These findings collectively support blockade of ASICs as a potential therapeutic strategy for PD. Further studies focusing on the effects of application of ASIC inhibitors to patients with PD are necessary to accurately define the specific roles of ASICs in this neurodegenerative disorder.

\section{ASICs and Huntington's disease}

Huntington's disease (HD) is a rare, progressive and fatal hereditary neurodegenerative disease characterized by movement and personality disorders and progressive cognitive decline [56], for which no completely effective treatments in human patients are currently available. A common and widely reported phenomenon of energy metabolism impairment in HD is accumulation of lactic acid in the CNS and possible subsequent acidosis in both animal models and human patients [57, 58]. Wong et al. [59] showed that the amiloride derivative, benzamil, a potent blocker of epithelial sodium channels, significantly 
decreases huntingtin-polyglutamine (htt-polyQ) aggregation in vitro. The therapeutic effect of benzamil was confirmed in the R6/2 animal model of HD. Administration of benzamil additionally ameliorated inhibition of ubiquitin-proteasome system (UPS) activity, promoting degradation of soluble htt-polyQ specifically in its pathological range. Furthermore, blockage of activity and/or expression of ASICla via RNA interference enhanced UPS activity and reduced htt-
polyQ aggregation in the striatum of R6/2 model mice.

The results suggest that ASICs play a pivotal role in the polyQ aggregating process and pathogenesis of $\mathrm{HD}$, and may therefore present an effective therapeutic target for progressive HD and other polyQ-related disorders. However, it is essential to establish the validity of ASIC inhibitors as HD treatment agents in preliminary preclinical studies.

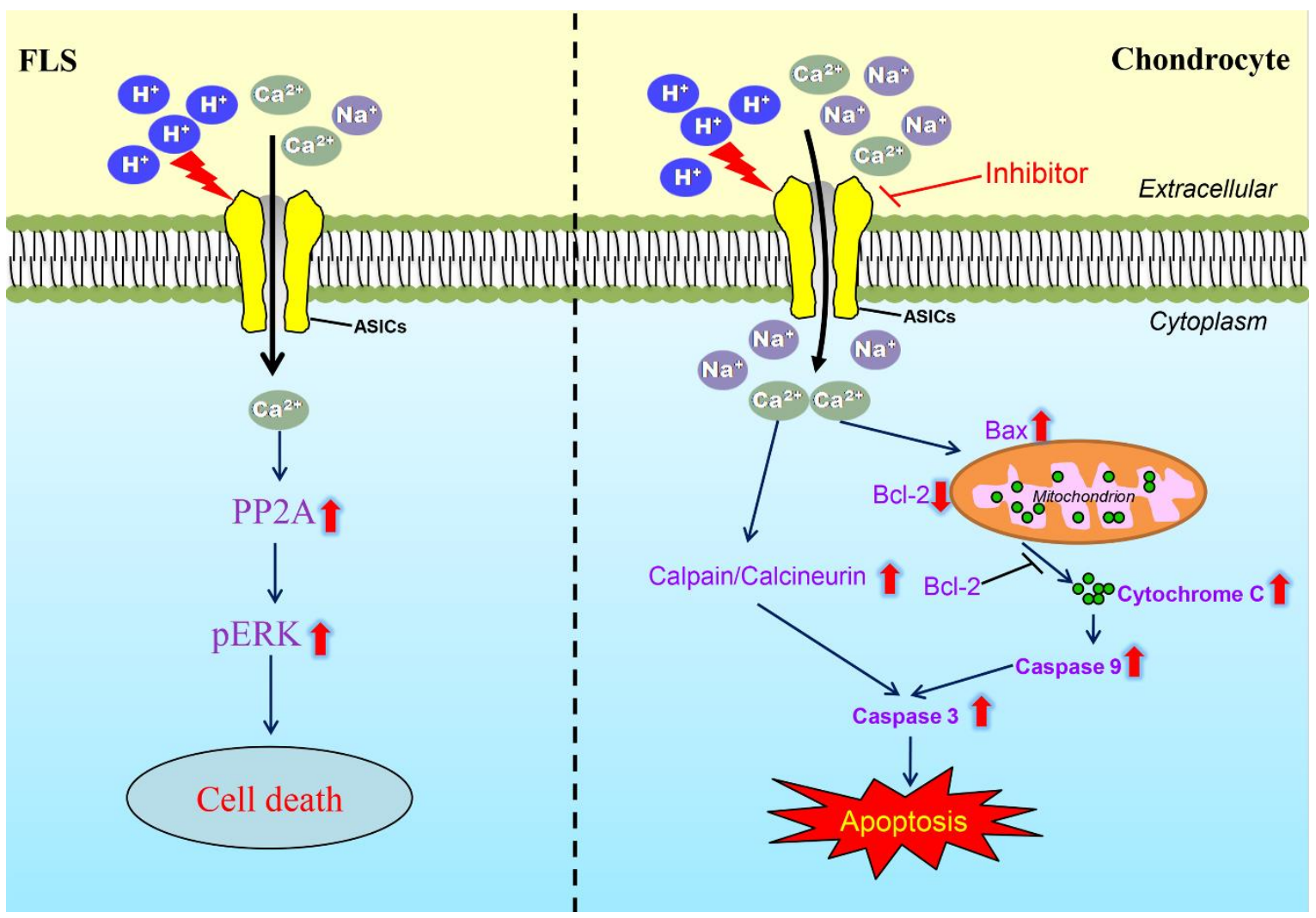

Figure 2. Roles of ASICs in arthritis fibroblast-like synoviocytes and chondrocytes. ASICs were activated by extracellular low $\mathrm{pH}$ to positively regulate $\left[\mathrm{Ca}^{2+}\right]_{i}$, inducing activation of PP2A and pERK to mediate cell death in FLS. Simultaneously, activated ASICs triggered intracellular $\mathrm{Ca}^{2+}$ accumulation in articular chondrocytes and subsequently upregulated Calpain, Calcineurin, Bax, Cytochrome c and Caspase 3/9, ultimately leading to chondrocyte apoptosis in arthritis.

\section{ASICs and Intervertebral Disc Degeneration}

Intervertebral disc degeneration (IVDD) is characterized by chronic excessive destruction of the extracellular matrix (ECM), leading to low back pain [60,61]. Although disc cell death through apoptosis is closely associated with development of IVDD, the underlying mechanisms are not fully elucidated. Under hypoxic conditions, disc cell metabolism is partially anaerobic, resulting in high concentrations of lactic acid and an acidic environment that is enhanced by the presence of cytokines [62, 63]. Matrix acidity has a potentially negative effect on gene expression, proliferation and viability of disc cells [64]. A recent study reported a significant increase in ASIC1 and ASIC4-positive cells in the annulus fibrosus of degenerated IVD and marked 
upregulation of ASIC1, ASIC2 and ASIC3 in the nucleus pulposus [30]. More importantly, $\mathrm{Li}$ et al. [65] demonstrated that acid-induced $\left[\mathrm{Ca}^{2+}\right]_{i}$ elevation via ASIC1a is involved in endplate chondrocyte apoptosis. Moreover, inhibition of ASIC1a using psalmotoxin 1 (PcTx1) or specific small interfering RNA (siRNA) suppressed acid-induced apoptosis and elevation of $\left[\mathrm{Ca}^{2+}\right]_{\mathrm{i}}$ in endplate chondrocytes of IVDs. Another recent study by Yuan et al. [66] demonstrated that ASIC1a activation by extracellular acid induces ECM metabolism via increasing matrix metalloproteinases activity and expression through the nuclear factor- $\kappa \mathrm{B}(\mathrm{NF}-\kappa \mathrm{B})$ signaling pathway in rat endplate chondrocytes.

These results provide evidence that the effects of ASIC1a on IVDD are attributable to its role in modulating cell apoptosis and ECM synthesis. However, gaps still exist in unraveling the precise regulatory mechanisms associating IVDD with different isoforms of ASICs. While these findings suggest considerable promise for inhibition of ASICs as a unique strategy for treatment of IVDD, further research is warranted to determine the biological role of ASICs in IVDD in vitro as well as in vivo.

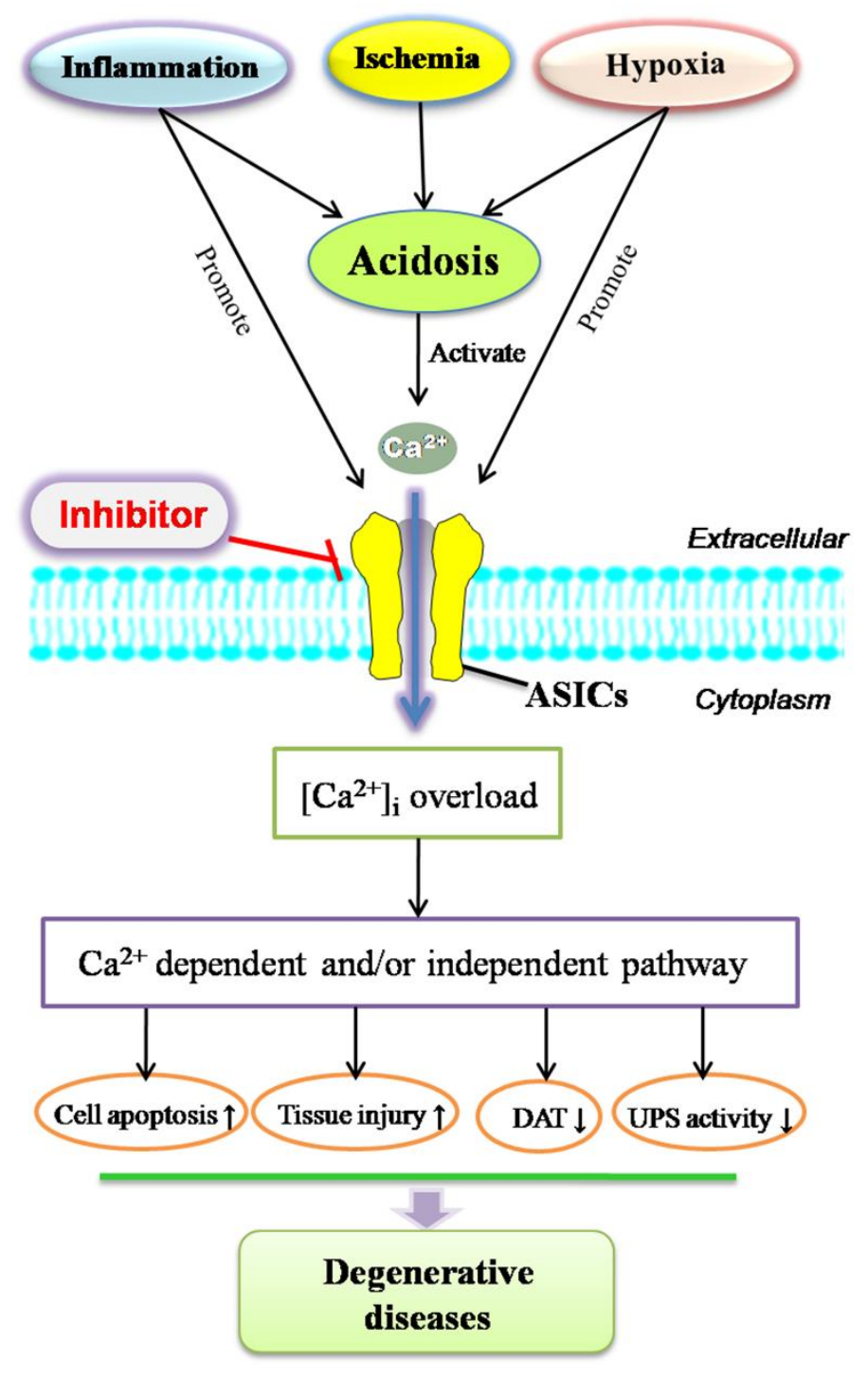

Figure 3. Schematic diagram summarizing the involvement of ASICs in cellular functions. Ischemia, inflammation and hypoxia give rise to tissue acidosis. ASICs are activated by extracellular $\mathrm{H}^{+}$and mediate $\mathrm{Ca}^{2+}$ influx. $\left[\mathrm{Ca}^{2+}\right]_{i}$ overload in various cell via ASICs leads to the development of degenerative diseases. 
Table 2. The roles of ASICs in degenerative diseases

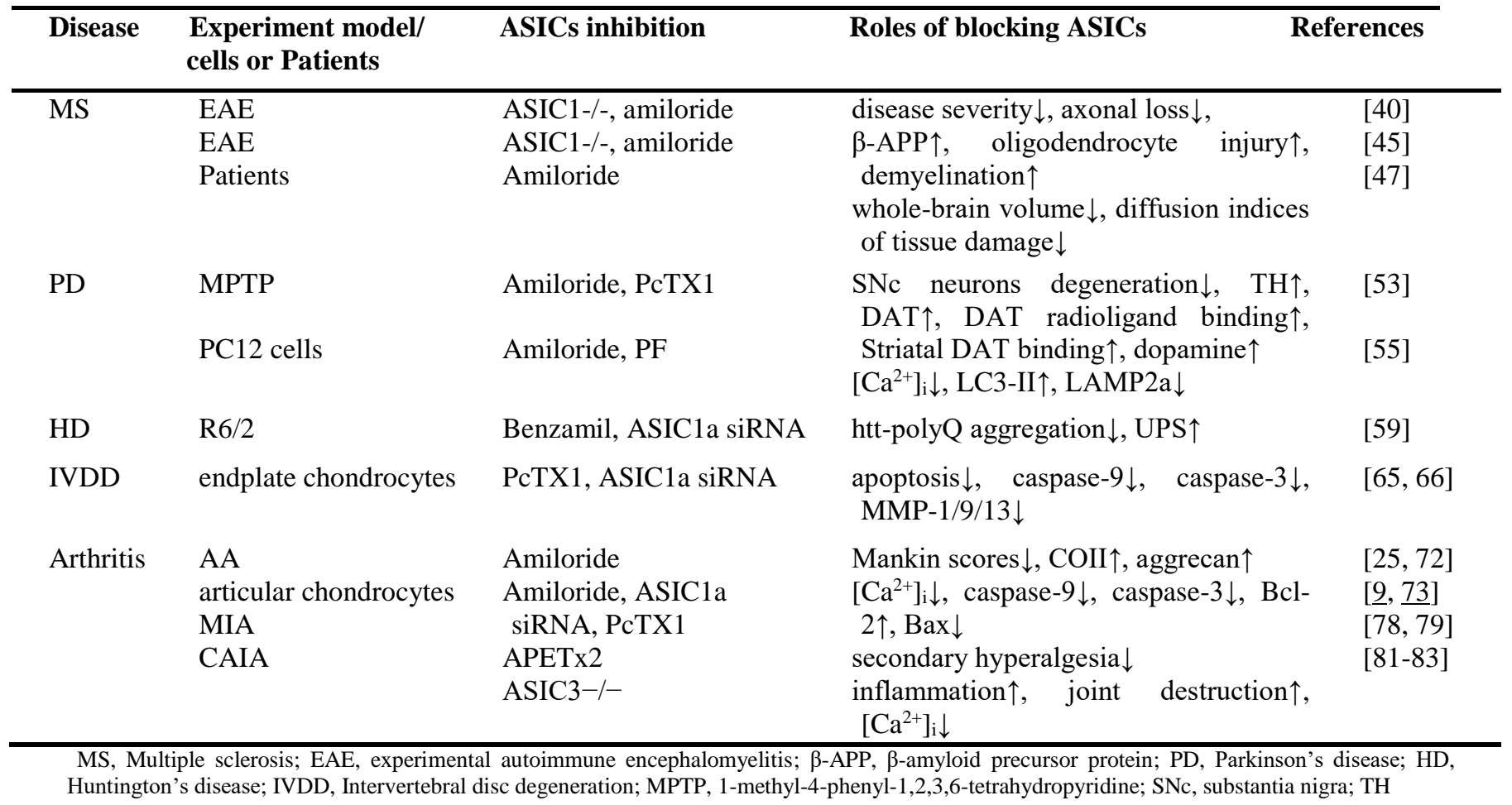

\section{ASICs and Arthritis}

Arthritis is inflammation of one or more joints resulting in cartilage and bone destruction. The main symptoms are joint pain, swelling and stiffness that are typically exacerbated with age. The most common types of arthritis are osteoarthritis (OA) and rheumatoid arthritis (RA) [67, 68]. Articular cartilage, a thick and highly hydrated biological soft tissue that lines the surfaces of bones in diarthrodial joints such as the knee, is critical for physiological mobility [69]. Under normal conditions, cartilage homeostasis is maintained by the balance between synthesis and degradation of ECM components, including type II collagen and aggrecan, the most abundant proteoglycan in articular chondrocytes [70]. However, in arthritis states, disruption of the matrix equilibrium leads to progressive loss of cartilage tissue and apoptosis of cells. Interestingly, matrix turnover is influenced by changes in chondrocytes exposed to extracellular acidosis [71]. Recently, to determine the potential involvement of ASICs in cartilage injury, our group tested the effects of amiloride both in vitro and in vivo [72]. We observed a significant increase in intracellular calcium in articular chondrocytes exposed to extracellular $\mathrm{pH}$ 6.0. Amiloride diminished this increase in $\left[\mathrm{Ca}^{2+}\right]_{\mathrm{i}}$ and attenuated acid-induced articular chondrocyte injury. In addition, amiloride induced a significant decrease in Mankin scores, but increased type II collagen and aggrecan mRNA and protein expression in articular cartilage in adjuvant arthritis (AA) rats. Blockade of ASIC1a with PcTX1 or specific siRNA inhibited acid-induced osteoclast differentiation and bone resorption via regulating activation of the transcription factor, nuclear factor of activated T cells c1 [10]. Similarly, the ASIC1a-specific blocker PcTX venom attenuated acid-induced articular chondrocyte damage [25]. Furthermore, blocking ASICs markedly decreased calpain and calcineurin expression levels as well as caspase-3/9 activity, and led to recovery of mitochondrial membrane potential via regulation of B-cell lymphoma-2 family gene expression in acid-induced chondrocytes [9, 73]. A more recent study reported that interleukin- 6 promotes acid-induced articular chondrocyte apoptosis to a significant extent by activating the JAK2/STAT3 and MAPK/NF- $\mathrm{BB}$ signaling pathways, resulting in upregulation of ASIC1 a expression and function [4].

Hidden chronic pain is one of the prominent clinical features of arthritis. Although significant progress has been made in terms of elucidating the mechanisms of joint pain at the cellular and molecular level, the data are insufficient to facilitate the development of more effective treatments for arthritic pain. Proton $\left(\mathrm{H}^{+}\right)$accumulation in 
tissues was recently shown to directly cause algesia and hyperalgesia. ASIC3, a main acid receptor, plays a key role in acid-induced injury and pain [74]. Babinski et al. [75] reported that ASIC3 is activated in the collageninduced arthritis pain model, which was also verified in AA [76]. Moreover, inflammation led to a decline in $\mathrm{pH}$, and in turn, activation of ASIC 3 on primary afferent fibers innervating the knee joint and generation of central pain hypersensitivity. ASIC3 plays an important role in secondary hyperalgesia of the paw, but has a weak influence on primary hyperalgesia [74, 76, 77]. A recent study demonstrated that weight distribution asymmetry and secondary hyperalgesia are inhibited by continuous intra-articular injection of APETx2 through attenuating ASIC3 upregulation in knee joint afferents in a monoiodoacetate-induced OA model [78]. Furthermore, Deval et al. [79] showed that knockdown of ASIC3 with siRNA and APETx2, a specific blocker of ASIC3, had markedly effective antinociceptive action against primary inflammation-induced hyperalgesia in rat.

Fibroblast-like synoviocytes (FLS) play a vital role in RA pathogenesis, and thus targeting FLS may ameliorate the clinical outcomes of inflammatory arthritis [80]. Interestingly, Kolker et al. [81] found a significantly lower increase in intracellular calcium and hyaluronan release in ASIC3 knockout FLS at pH 5.5, compared to control FLS. Unexpectedly, joint inflammation and destruction were significantly enhanced in ASIC3 knockout mice with collagen antibody-induced arthritis, compared to wild type (WT) mice. Moreover, FLS exposed to $\mathrm{pH} 6.0$ displayed enhanced cell death in the presence of IL-1, which was eliminated in ASIC3-/- FLS [82]. IL-1 $\beta$ upregulated ASIC3 mRNA and enhanced $\left[\mathrm{Ca}^{2+}\right]_{\mathrm{i}}, \mathrm{p}$-ERK, IL-6 and metalloproteinase mRNA as well as cell death in WT FLS exposed to $\mathrm{pH}$ 6.0. Inhibitors of $\left[\mathrm{Ca}^{2+}\right]_{\mathrm{i}}$ and ERK prevented cell death induced by acidic $\mathrm{pH}$ in combination with IL-1 $\beta$ in WT FLS [83]. Since ASIC3 itself is not $\mathrm{Ca}^{2+}$ permeable, the mechanism of ASIC3-mediated regulation of $\left[\mathrm{Ca}^{2+}\right]_{i}$ may be as follows: deletion of ASIC3 reduces $\mathrm{pH}$ sensitivity of ASICs, release of internal $\mathrm{Ca}^{2+}$ stores and secondary activation of other $\mathrm{Ca}^{2+}$-permeable channels [84-86]. These findings suggest that ASIC3 plays a protective role in inflammatory arthritis via inhibiting synovial proliferation, which reduces accumulation of inflammatory cytokines and subsequent joint damage (Fig.2).

ASIC1a may therefore promote articular chondrocyte apoptosis as well as osteoclast differentiation. Moreover, elevated levels of ASIC3 in the DRG contribute to arthritic pain, supporting inhibition of ASICs as a potential therapeutic strategy for arthritis. However, ASIC3 has also been shown to play an inhibitory role in synovial proliferation and subsequent accumulation of inflammatory mediators, indicative of a protective effect in joints. Although several investigators agree that ASICs are involved in the pathogenesis of arthritis, in light of the controversial evidence, the roles of these ion channels in arthritis require further investigation. Clarification of the precise roles of different ASIC subtypes in the pathogenesis of arthritis may aid in the identification of novel pharmacological targets (Table 2).

\section{Conclusion}

Acid-alkaline balance is an important factor for maintaining normal physiological activities. However, almost all types of pathological states, such as ischemia, inflammation, hypoxia and cancer, lead to variations in $\mathrm{pH}$. Recent research has focused on the roles of ion channels, such as $\mathrm{DEG} / \mathrm{ENaC}$, in diseases. In particular, given that ASICs serve as pivotal acid receptors, multiple lines of evidence support their involvement in the progression of tissue acidosis. Interestingly, this protein family is closely correlated with various degenerative diseases, supporting their potential value as therapeutic targets (Fig.3). The current study showed that amiloride is a classic inhibitor drug of the DEG/ENaC family, but not selective for ASICs. Although several specific ASIC blockers exist, such as PcTx1 and APETx2, their clinical utility remains to be determined. With the advances in biological and molecular techniques, improved knowledge on ASIC function and structure should facilitate the development of a suitable and specific inhibitor for clinical treatment of degenerative disorders. However, the current evidence regarding ASICs in degenerative diseases is relatively limited, the majority of which has been obtained using animal models or in vitro systems. Human studies with larger patient populations are required to accurately resolve the mechanisms of action of ASICs in the development of degenerative disorders, which may provide a rationale for developing effective therapeutic interventions targeting ASICs for preventing degenerative disease progression.

\section{Conflicts of interest}

The authors declare no conflicts of interest.

\section{Acknowledgements}

This project was supported by the National Science Foundation of China (No. 81271949, 30873080).

\section{References}

[1] Zuba-Surma EK, Wojakowski W, Madeja Z, Ratajczak MZ (2012). Stem Cells as a Novel Tool for Drug 
Screening and Treatment of Degenerative Diseases. Current Pharmaceutical Design, 18: 2644-56.

[2] Mawad D, Boughton EA, Boughton P, Lauto A (2012). Advances in hydrogels applied to degenerative diseases. Curr Pharm Des, 18: 2558-75.

[3] Jin C, Ye QH, Yuan FL, Gu YL, Li JP, Shi YH, et al. (2015). Involvement of acid-sensing ion channel 1alpha in hepatic carcinoma cell migration and invasion. Tumour Biol, 36: 4309-17.

[4] Zhou R, Wu X, Wang Z, Ge J, Chen F (2015). Interleukin-6 enhances acid-induced apoptosis via upregulating acid-sensing ion channel la expression and function in rat articular chondrocytes. Int Immunopharmacol, 29: 748-60.

[5] Uchiyama Y, Guttapalli A, Gajghate S, Mochida J, Shapiro IM, Risbud MV (2008). SMAD3 functions as a transcriptional repressor of acid-sensing ion channel 3 (ASIC3) in nucleus pulposus cells of the intervertebral disc. J Bone Miner Res, 23: 1619-28.

[6] Sun X, Zhao D, Li YL, Sun Y, Lei XH, Zhang JN, et al. (2013). Regulation of ASIC1 by Ca2+/calmodulindependent protein kinase II in human glioblastoma multiforme. Oncol Rep, 30: 2852-8.

[7] Berridge MJ, Bootman MD, Roderick HL (2003). Calcium signalling: dynamics, homeostasis and remodelling. Nat Rev Mol Cell Biol, 4: 517-29.

[8] Li CK, Meng L, Li X, Li DL, Jiang LH (2015). NonNMDAR neuronal $\mathrm{Ca} 2+$-permeable channels in delayed neuronal death and as potential therapeutic targets for ischemic brain damage. Expert Opinion on Therapeutic Targets, 19: 879-92.

[9] Rong C, Chen FH, Jiang S, Hu W, Wu FR, Chen TY, et al. (2012). Inhibition of acid-sensing ion channels by amiloride protects rat articular chondrocytes from acid-induced apoptosis via a mitochondrial-mediated pathway. Cell Biol Int, 36: 635-41.

[10] Li X, Xu RS, Jiang DL, He XL, Jin C, Lu WG, et al. (2013). Acid-sensing ion channel 1a is involved in acid-induced osteoclastogenesis by regulating activation of the transcription factor NFATc1. FEBS Lett, 587: 3236-42.

[11] Sun X, Cao YB, Hu LF, Yang YP, Li J, Wang F, et al. (2011). ASICs mediate the modulatory effect by paeoniflorin on alpha-synuclein autophagic degradation. Brain Res, 1396: 77-87.

[12] Lingueglia E (2007). Acid-sensing ion channels in sensory perception. J Biol Chem, 282: 17325-9.

[13] Leng T, Lin J, Cottrell JE, Xiong ZG (2013). Subunit and frequency-dependent inhibition of acid sensing ion channels by local anesthetic tetracaine. Mol Pain, 9: 27.

[14] Li X, Fei J, Lei Z, Liu K, Wu J, Meng T, et al. (2014). Chloroquine impairs visual transduction via modulation of acid sensing ion channel 1a. Toxicology letters, 228: 200-6.

[15] Zha XM (2013). Acid-sensing ion channels: trafficking and synaptic function. Mol Brain, 6: 1 .

[16] Swain SM, Bera AK (2013). Coupling of proton binding in extracellular domain to channel gating in acid-sensing ion channel. J Mol Neurosci, 51: 199-207.
Jasti J, Furukawa H, Gonzales EB, Gouaux E (2007). Structure of acid-sensing ion channel 1 at $1.9 \mathrm{~A}$ resolution and low $\mathrm{pH}$. Nature, 449: 316-23.

[18] Gonzales EB, Kawate T, Gouaux E (2009). Pore architecture and ion sites in acid-sensing ion channels and P2X receptors. Nature, 460: 599-604.

[19] Sherwood TW, Lee KG, Gormley MG, Askwith CC (2011). Heteromeric Acid-Sensing Ion Channels (ASICs) Composed of ASIC2b and ASIC1a Display Novel Channel Properties and Contribute to AcidosisInduced Neuronal Death. Journal of Neuroscience, 31: 9723-34.

[20] Gautam M, Benson CJ (2013). Acid-sensing ion channels (ASICs) in mouse skeletal muscle afferents are heteromers composed of ASIC1a, ASIC2, and ASIC3 subunits. FASEB J, 27: 793-802.

[21] Ugawa S, Ueda T, Ishida Y, Nishigaki M, Shibata Y, Shimada S (2002). Amiloride-blockable acid-sensing ion channels are leading acid sensors expressed in human nociceptors. Journal of Clinical Investigation, 110: 1185-90.

[22] G.R. Dubé*, A. Elagoz , Mangat H (2009). Acid sensing ion channels and acid nociception. Current Pharmaceutical Design, 15: 1750-66.

[23] Benarroch EE (2014). Acid-sensing cation channels: structure, function, and pathophysiologic implications. Neurology, 82: 628-35.

[24] Della Vecchia MC, Rued AC, Carattino MD (2013). Gating transitions in the palm domain of ASIC1a. J Biol Chem, 288: 5487-95.

[25] Yuan FL, Chen FH, Lu WG, Li X, Wu FR, Li JP, et al. (2010). Acid-sensing ion channel 1a mediates acidinduced increases in intracellular calcium in rat articular chondrocytes. Mol Cell Biochem, 340: 1539.

[26] Weng JY, Lin YC, Lien CC (2010). Cell type-specific expression of acid-sensing ion channels in hippocampal interneurons. J Neurosci, 30: 6548-58.

[27] Duan B, Wang YZ, Yang T, Chu XP, Yu Y, Huang Y, et al. (2011). Extracellular spermine exacerbates ischemic neuronal injury through sensitization of ASIC1a channels to extracellular acidosis. J Neurosci, 31: 2101-12.

[28] Kuduk SD, Chang RK, Wai JM, Di Marco CN, Cofre V, DiPardo RM, et al. (2009). Amidine derived inhibitors of acid-sensing ion channel-3 (ASIC3). Bioorg Med Chem Lett, 19: 4059-63.

[29] Vega R, Rodriguez U, Soto E (2009). Acid-sensing ionic-channel functional expression in the vestibular endorgans. Neurosci Lett, 463: 199-202.

[30] Cuesta A, Del Valle ME, Garcia-Suarez O, Vina E, Cabo R, Vazquez G, et al. (2014). Acid-sensing ion channels in healthy and degenerated human intervertebral disc. Connect Tissue Res, 55: 197-204.

[31] Baconguis I, Gouaux E (2012). Structural plasticity and dynamic selectivity of acid-sensing ion channelspider toxin complexes. Nature, 489: 400-5.

[32] Bhattacharya P, Pandey AK, Paul S, Patnaik R (2012). Neuroprotective potential of Piroxicam in cerebral ischemia: an in silico evaluation of the hypothesis to 
explore its therapeutic efficacy by inhibition of aquaporin-4 and acid sensing ion channella. Med Hypotheses, 79: 352-7.

[33] Ziemann AE, Allen JE, Dahdaleh NS, Drebot II, Coryell MW, Wunsch AM, et al. (2009). The Amygdala Is a Chemosensor that Detects Carbon Dioxide and Acidosis to Elicit Fear Behavior. Cell, 139: 1012-21.

[34] Calavia MG, Montano JA, Garcia-Suarez O, Feito J, Guervos MA, Germana A, et al. (2010). Differential localization of Acid-sensing ion channels 1 and 2 in human cutaneus pacinian corpuscles. Cell Mol Neurobiol, 30: 841-8.

[35] Harding AMS, Kusama N, Hattori T, Gautam M, Benson CJ (2014). ASIC2 Subunits Facilitate Expression at the Cell Surface and Confer Regulation by PSD-95. Plos One, 9: e93797.

[36] Feldman DH, Horiuchi M, Keachie K, McCauley E, Bannerman P, Itoh A, et al. (2008). Characterization of acid-sensing ion channel expression in oligodendrocyte-lineage cells. Glia, 56: 1238-49.

[37] Hesselager M, Timmermann DB, Ahring PK (2004). $\mathrm{pH}$ Dependency and desensitization kinetics of heterologously expressed combinations of acidsensing ion channel subunits. J Biol Chem, 279: 11006-15.

[38] Wu WL, Cheng CF, Sun WH, Wong CW, Chen CC (2012). Targeting ASIC3 for pain, anxiety, and insulin resistance. Pharmacol Ther, 134: 127-38.

[39] Chu XP, Miesch J, Johnson M, Root L, Zhu XM, Chen D, et al. (2002). Proton-gated channels in PC12 cells. J Neurophysiol, 87: 2555-61.

[40] Friese MA, Craner MJ, Etzensperger R, Vergo S, Wemmie JA, Welsh MJ, et al. (2007). Acid-sensing ion channel-1 contributes to axonal degeneration in autoimmune inflammation of the central nervous system. Nat Med, 13: 1483-9.

[41] Jana A, Pahan K (2010). Sphingolipids in multiple sclerosis. Neuromolecular Med, 12: 351-61.

[42] Friese MA, Schattling B, Fugger L (2014). Mechanisms of neurodegeneration and axonal dysfunction in multiple sclerosis. Nat Rev Neurol, 10: 225-38.

[43] Chu XP, Xiong ZG (2012). Physiological and pathological functions of acid-sensing ion channels in the central nervous system. Curr Drug Targets, 13: 263-71.

[44] Lubetzki C, Stankoff B (2014). Demyelination in multiple sclerosis. Handb Clin Neurol, 122: 89-99.

[45] Vergo S, Craner MJ, Etzensperger R, Attfield K, Friese MA, Newcombe J, et al. (2011). Acid-sensing ion channel 1 is involved in both axonal injury and demyelination in multiple sclerosis and its animal model. Brain, 134: 571-84.

[46] Boiko N, Kucher V, Eaton BA, Stockand JD (2013). Inhibition of neuronal degenerin/epithelial $\mathrm{Na}+$ channels by the multiple sclerosis drug 4aminopyridine. J Biol Chem, 288: 9418-27.

[47] Arun T, Tomassini V, Sbardella E, de Ruiter MB, Matthews L, Leite MI, et al. (2013). Targeting ASIC1
[61] Fontana G, See E, Pandit A (2015). Current trends in biologics delivery to restore intervertebral disc anabolism. Adv Drug Deliv Rev, 84: 146-58.

[62] Sakai D, Grad S (2015). Advancing the cellular and

in primary progressive multiple sclerosis: evidence of neuroprotection with amiloride. Brain, 136: 106-15. L. B, SB. M, PP. B, L. F, R. F, L. M, et al. (2007). Association between the ACCN1 gene and multiple sclerosis in Central East. PLoS One, 5: e480.

Parish CL, Thompson LH (2014). Modulating Wnt signaling to improve cell replacement therapy for Parkinson's disease. J Mol Cell Biol, 6: 54-63.

Rangel-Barajas C, Coronel I, Floran B (2015). Dopamine Receptors and Neurodegeneration. Aging Dis, 6: 349-68.

Venderova K, Park DS (2012). Programmed cell death in Parkinson's disease. Cold Spring Harb Perspect Med, 2: a009365.

Picloplichko VI, Dani JA (2006). Acid-sensitive ionic channels in midbrain dopamine neurons are sensitive to ammonium, which may contribute to hyperammonemia damage. Proceedings of the National Academy of Sciences of the United States of America, 103: 11376-80.

Arias RL, Sung ML, Vasylyev D, Zhang MY, Albinson $\mathrm{K}$, Kubek K, et al. (2008). Amiloride is neuroprotective in an MPTP model of Parkinson's disease. Neurobiol Dis, 31: 334-41.

Joch M, Ase AR, Chen CX, MacDonald PA, Kontogiannea M, Corera AT, et al. (2007). Parkinmediated monoubiquitination of the PDZ protein PICK1 regulates the activity of acid-sensing ion channels. Mol Biol Cell, 18: 3105-18. al. (2010). Paeoniflorin, a potent natural compound, protects $\mathrm{PC} 12$ cells from MPP+ and acidic damage via autophagic pathway. J Ethnopharmacol, 131: 122-9.

Agostinho LA, Dos Santos SR, Alvarenga RM, Paiva CL (2013). A systematic review of the intergenerational aspects and the diverse genetic profiles of Huntington's disease. Genet Mol Res, 12: 1974-81.

Tsz M. Tsang, Ben Woodman, Gerard A. Mcloughlin, Julian L. Griffin, Sarah J. Tabrizi, Gillian P. Bates, et al. (2006). Metabolic Characterization of the R6/2 Transgenic Mouse Model of Huntington's Disease by High-Resolution MAS ${ }^{1} \mathrm{H}$ NMR Spectroscopy. Journal of Proteome Research, 5: 483-92.

Josefsen K, Nielsen SM, Campos A, Seifert T, Hasholt L, Nielsen JE, et al. (2010). Reduced gluconeogenesis and lactate clearance in Huntington's disease. Neurobiol Dis, 40: 656-62.

Wong HK, Bauer PO, Kurosawa M, Goswami A, Washizu C, Machida Y, et al. (2008). Blocking acidsensing ion channel 1 alleviates Huntington's disease pathology via an ubiquitin-proteasome systemdependent mechanism. Hum Mol Genet, 17: 3223-35. Risbud MV, Shapiro IM (2014). Role of cytokines in intervertebral disc degeneration: pain and disc content. Nat Rev Rheumatol, 10: 44-56. 
molecular therapy for intervertebral disc disease. Adv Drug Deliv Rev, 84: 159-71.

[63] Tetlow LC, Adlam DJ, Woolley DE (2001). Matrix metalloproteinase and proinflammatory cytokine production by chondrocytes of human osteoarthritic cartilage: associations with degenerative changes. Arthritis Rheum, 44: 585-94.

[64] Wuertz K, Godburn K, Iatridis JC (2009). MSC response to $\mathrm{pH}$ levels found in degenerating intervertebral discs. Biochem Biophys Res Commun, 379: 824-9.

[65] Li X, Wu FR, Xu RS, Hu W, Jiang DL, Ji C, et al. (2014). Acid-sensing ion channel 1a-mediated calcium influx regulates apoptosis of endplate chondrocytes in intervertebral discs. Expert Opin Ther Targets, 18: 114.

[66] Yuan FL, Zhao MD, Jiang DL, Jin C, Liu HF, Xu MH, et al. (2015). Involvement of acid-sensing ion channel 1a in matrix metabolism of endplate chondrocytes under extracellular acidic conditions through NFkappaB transcriptional activity. Cell Stress Chaperones, 27:97-104.

[67] Scott DL, Wolfe F, Huizinga TW (2010). Rheumatoid arthritis. Lancet, 376: 1094-108.

[68] Musumeci G, Szychlinska MA, Mobasheri A (2015). Age-related degeneration of articular cartilage in the pathogenesis of osteoarthritis: molecular markers of senescent chondrocytes. Histol Histopathol, 30: 1-12.

[69] Ge Z, Li C, Heng BC, Cao G, Yang Z (2012). Functional biomaterials for cartilage regeneration. $\mathrm{J}$ Biomed Mater Res A, 100: 2526-36.

[70] Lee AS, Ellman MB, Yan DY, Kroin JS, Cole BJ, van Wijnen AJ, et al. (2013). A current review of molecular mechanisms regarding osteoarthritis and pain. Gene, 527: 440-7.

[71] Razaq S, Wilkins RJ, Urban JP (2003). The effect of extracellular $\mathrm{pH}$ on matrix turnover by cells of the bovine nucleus pulposus. Eur Spine J, 12: 341-9.

[72] Yuan FL, Chen FH, Lu WG, Li X, Li JP, Li CW, et al. (2010). Inhibition of acid-sensing ion channels in articular chondrocytes by amiloride attenuates articular cartilage destruction in rats with adjuvant arthritis. Inflamm Res, 59: 939-47.

[73] $\mathrm{Hu}$ W, Chen FH, Yuan FL, Zhang TY, Wu FR, Rong C, et al. (2012). Blockade of acid-sensing ion channels protects articular chondrocytes from acid-induced apoptotic injury. Inflamm Res, 61: 327-35.

[74] Ikeuchi M, Kolker SJ, Burnes LA, Walder RY, Sluka KA (2008). Role of ASIC3 in the primary and secondary hyperalgesia produced by joint inflammation in mice. Pain, 137: 662-9.
[75]

Babinski K, Le KT, Seguela P (1999). Molecular cloning and regional distribution of a human proton receptor subunit with biphasic functional properties. J Neurochem, 72: 51-7.

[76] Ikeuchi M, Kolker SJ, Sluka KA (2009). Acid-sensing ion channel 3 expression in mouse knee joint afferents and effects of carrageenan-induced arthritis. J Pain, 10: 336-42.

[77] Yen YT, Tu PH, Chen CJ, Lin YW, Hsieh ST, Chen CC (2009). Role of acid-sensing ion channel 3 in subacute-phase inflammation. Mol Pain, 5: 1.

[78] Izumi M, Ikeuchi M, Ji Q, Tani T (2012). Local ASIC3 modulates pain and disease progression in a rat model of osteoarthritis. Journal of Biomedical Science, 19: 77.

[79] Deval E, Noel J, Lay N (2008). ASIC3, a sensor of acidic and primary inflammatory pain. The EMBO Journal, 27: 3047-55.

[80] Zong M, Lu T, Fan S, Zhang H, Gong R, Sun L, et al. (2015). Glucose-6-phosphate isomerase promotes the proliferation and inhibits the apoptosis in fibroblastlike synoviocytes in rheumatoid arthritis. Arthritis Res Ther, 17: 100.

[81] Kolker SJ, Walder RY, Usachev Y, Hillman J, Boyle DL, Firestein GS, et al. (2010). Acid-sensing ion channel 3 expressed in type B synoviocytes and chondrocytes modulates hyaluronan expression and release. Ann Rheum Dis, 69: 903-9.

[82] Sluka KA, Rasmussen LA, Edgar MM, O'Donnell JM, Walder RY, Kolker SJ, et al. (2013). Acid-sensing ion channel 3 deficiency increases inflammation but decreases pain behavior in murine arthritis. Arthritis Rheum, 65: 1194-202.

[83] Gong W, Kolker SJ, Usachev Y, Walder RY, Boyle DL, Firestein GS, et al. (2014). Acid-sensing ion channel 3 decreases phosphorylation of extracellular signalregulated kinases and induces synoviocyte cell death by increasing intracellular calcium. Arthritis Res Ther, 16: R121.

[84] Xiong ZG, Xu TL (2012). The role of ASICS in cerebral ischemia. Wiley Interdiscip Rev Membr Transp Signal, 1: 655-62.

[85] Lu Y, Whiteis CA, Sluka KA, Chapleau MW, Abboud FM (2013). Responses of glomus cells to hypoxia and acidosis are uncoupled, reciprocal and linked to ASIC3 expression: selectivity of chemosensory transduction. J Physiol, 591: 919-32.

[86] Xu TL, Xiong ZG (2007). Dynamic regulation of acidsensing ion channels by extracellular and intracellular modulators. Curr Med Chem, 14: 1753-63. 